\title{
Zur Erforschung der Dorfkirchen in Mitteldeutschland Bemerkungen anlässlich einiger Neuerscheinungen
}

\author{
von \\ ENNO BÜNZ
}

\begin{abstract}
„Nur unsere Dorfkirchen stellen sich uns vielfach als die Träger unserer ganzen Geschichte dar, und die Berührung der Jahrhunderte untereinander zur Erscheinung bringend, besitzen und äußern sie den Zauber historischer Kontinuität“ (Fontane) $)^{1}$
\end{abstract}

Von Bedeutung, Funktion und Entwicklung der Pfarrkirche als der wohl bedeutendsten Schnittstelle von Kirche und Welt haben gerade in den letzten beiden Jahrzehnten zahlreiche landesgeschichtliche Untersuchungen ein anschauliches Bild gezeichnet. ${ }^{2}$ In der Ausstellung „Alltag und Frömmigkeit am Vorabend der Reformation in Mitteldeutschland“, die von 2013 bis 2015 in gewandelter Gestalt in Mühlhausen, Leipzig und Magdeburg zu sehen war, stand konsequent die Pfarrei als die zentrale Seelsorgeinstitution und die kirchliche Alltagserfahrung der Christen im Mittelpunkt. ${ }^{3}$ Diese publikumswirksame Präsentation kann aber nicht darüber hinwegtäuschen, dass die Entwicklung der Pfarrorganisation und des Pfarreilebens in Mitteldeutschland noch vielfältiger Erforschung bedarf. Auch für Sachsen besteht hier noch ein erheblicher Nachholbedarf, obschon Walter Schlesinger mit seiner zweibändigen Kirchengeschichte Sachsens im Mittelalter zumindest bis ca. 1300 für die drei Bistümer Meißen, Merseburg und Naumburg - also für einen Raum, der weit über das Gebiet des heuti-

1 Theodor Fontane, Vor dem Sturm. Roman aus dem Winter 1812 auf 13, Bd. 1-2, hrsg. von Christine Hehle (Große Brandenburger Ausgabe. Das erzählerische Werk 1), Berlin 2011, S. 43.

2 Zahlreiche weiterführende Hinweise in: Wolfgang Petke, Die Pfarrei. Ein Institut von langer Dauer als Forschungsaufgabe, in: Enno Bünz/Klaus-Joachim LorenzenSchmidt (Hg.), Klerus, Kirche, Frömmigkeit im mittelalterlichen Schleswig-Holstein (Studien zur Wirtschafts- und Sozialgeschichte Schleswig-Holsteins 41), Neumünster 2006, S. 17-49; EnNo BünZ, Die mittelalterliche Pfarrei in Deutschland. Neue Forschungstendenzen und -ergebnisse, in: Nathalie Kruppa (Hg.), Pfarreien im Mittelalter. Deutschland, Polen, Tschechien und Ungarn im Vergleich (Veröffentlichungen des Max-Planck-Instituts für Geschichte 238 = Studien zur Germania Sacra 32), Göttingen 2008, S. 27-66 und die Beiträge in Enno Bünz/Gerhard Fouquet (Hg.), Die Pfarrei im späten Mittelalter (Vorträge und Forschungen 77), Ostfildern 2013.

3 Hartmut Kühne/Enno Bünz/Thomas T. MülleR (Hg.), Alltag und Frömmigkeit am Vorabend der Reformation in Mitteldeutschland. Katalog zur Ausstellung „Umsonst ist der Tod“, Petersberg 2013. - Viele neue Forschungsergebnisse bieten die Referate einer zur Vorbereitung der Ausstellung veranstalteten Tagung: Alltag und Frömmigkeit am Vorabend der Reformation in Mitteldeutschland. Wissenschaftlicher Begleitband zur Ausstellung: EnNo Bünz/HaRTmut KüHne (Hg.), „Umsonst ist der Tod“ (Schriften zur sächsischen Geschichte und Volkskunde), Leipzig 2015 (im Druck). 
gen Freistaates Sachsen hinausreicht - wichtige Grundlagen gelegt hat, auf denen für das späte Mittelalter und darüber hinaus aufgebaut werden kann. ${ }^{4}$ Die Pfarrorganisation dieser drei Bistümer am Ende des Mittelalters ist mittlerweile auch kartografisch präzise dargestellt worden. ${ }^{5}$ In den letzten Jahren haben vor allem die kirchlichen Verhältnisse in den größeren Städten Sachsens Aufmerksamkeit gefunden. ${ }^{6}$ Für die vorreformatorischen Dorfpfarreien werden schon aufgrund der Quellenlage zumeist zwar keine historischen Einzelfallstudien möglich sein, doch bieten sie aus regionaler Perspektive durchaus noch viele Ansatzpunkte für Untersuchungen, die mehrere Pfarreien vergleichend betrachten oder systematisch bestimmte Quellentypen auswerten. ${ }^{7}$

4 Walter Schlesinger, Kirchengeschichte Sachsens im Mittelalter, 2 Bde. (Mitteldeutsche Forschungen 27/1-2), Köln/Graz 1962, ${ }^{2} 1983$, hier Bd. 1, S. 143-214 über die ältesten Pfarrkirchen, und Bd. 2, S. 351-425 über die neuen Pfarrkirchen in Stadt und Land.

5 Karlheinz Blaschke/Walther Haupt/Heinz Wiessner, Die Kirchenorganisation in den Bistümern Meissen, Merseburg und Naumburg um 1500, Weimar 1969; KARLHeinz Blaschke/Manfred Kobuch, Kirchenorganisation um 1500. Karte 1:400.000 und Beiheft (Atlas zur Geschichte und Landeskunde von Sachsen, E II 1), Dresden/ Leipzig 2008.

6 Alexandra-Kathrin Stanislaw-Kemenah, Kirche, geistliches Leben und Schulwesen im Spätmittelalter, in: Karlheinz Blaschke/Uwe John (Hg.), Geschichte der Stadt Dresden, Bd. 1: Von den Anfängen bis zum Ende des Dreißigjährigen Krieges, Stuttgart 2005, S. 198-246 und S. 662-673; Anja Zschornak, Stadt und Kirche im spätmittelalterlichen Kamenz, in: Neues Lausitzisches Magazin N. F. 13 (2010), S. 31-54 (Zusammenfassung der ungedruckten Leipziger Magisterarbeit); Christian SPeER, Frömmigkeit und Politik. Städtische Eliten in Görlitz zwischen 1300 und 1550 (Hallische Beiträge zur Geschichte des Mittelalters und der Frühen Neuzeit 8), Berlin 2011; UwE Fiedler/Hendrik Thoss/Enno Bünz (Hg.), Des Himmels Fundgrube. Chemnitz und das sächsisch-böhmische Gebirge im 15. Jahrhundert, Chemnitz 2012; Julia KaHLEYss, Die Bürger von Zwickau und ihre Kirche. Kirchliche Institutionen und städtische Frömmigkeit im späten Mittelalter (Schriften zur sächsischen Geschichte und Volkskunde 45), Leipzig 2013; Dirk Martin MüTze, Das Augustiner-Chorherrenstift St. Afra in Meißen (1205-1539), Phil. Diss. (masch.) 2012 (erscheint 2015 in den „Schriften zur sächsischen Geschichte und Volkskunde"); Ulrike Siewert (Hg.), Die Stadtpfarrkirchen Sachsens im Mittelalter und in der Frühen Neuzeit (Bausteine aus dem Institut für sächsische Geschichte und Volkskunde 27), Dresden 2013; EnNo Bünz/Armin Kohnle (Hg.), Das religiöse Leipzig. Stadt und Glauben vom Mittelalter bis zur Gegenwart (Quellen und Forschungen zur Geschichte der Stadt Leipzig 6), Leipzig 2013; Petr Hrachovec, Die Zittauer und ihre Kirchen (ca. 1300-1600), Phil. Diss. (masch.) Karlsuniversität Prag 2014 (in deutscher Sprache!).

7 Aus der Fülle meiner einschlägigen Arbeiten nenne ich hier nur die mit einem Bezug zu Sachsen: EnNo BünZ, Bamberg - Regensburg - Naumburg. Das Vogtland im Spannungsfeld mittelalterlicher Kirchengeschichte, in: Herbergen der Christenheit. Jahrbuch für deutsche Kirchengeschichte 28/29 (2004/05), S. 27-54; Ders., „Neun Teufel, die den Pfarrer quälen“. Zum Alltag in den mittelalterlichen Pfarreien der Oberlausitz, in: Lars-Arne Dannenberg/Dietrich Scholze (Hg.), Stätten und Stationen religiösen Wirkens. Studien zur Kirchengeschichte der zweisprachigen Oberlausitz (Schriften des Sorbischen Instituts. Spisy Serbskeho instituta 48), Bautzen 2009, S. 19-54; Ders., Die Bauern und ihre Kirche. Zum Bauboom auf dem Land um 1500, in: Carola Fey/Steffen Krieb (Hg.), Adel und Bauern in der Gesellschaft des Mittelalters. Internationales Kolloquium zum 65. Geburtstag von Werner Rösener (Studien und Texte zur Geistesund Sozialgeschichte des Mittelalters 6), Korb 2012, S. 223-248; Ders., Die Römische Kurie und Sachsen im späten Mittelalter. Mit einer Zusammenstellung der Benefizien 
Aus landesgeschichtlicher Perspektive wären vor allem breiter angelegte Forschungen zu den Patronatsverhältnissen und zur Bedeutung der Inkorporationen, zur Einkommenssituation der Pfarreien (bzw. der Pfarrer) und zum Stiftungswesen, aber auch zur Liturgie und zum Frömmigkeitsleben wünschenswert.

Nun ist die Pfarrkirche allerdings eine Institution, die nicht nur den Landes- oder Kirchenhistoriker angeht, sondern auch Vertreter anderer historisch arbeitender Disziplinen. Vor allem von den Leistungen der Kunstgeschichte, Bauforschung und Archäologie soll im Folgenden die Rede sein. Im Gegensatz zu den Städten stellt die Pfarrkirche im Dorf in den allermeisten Fällen das einzige Bauwerk dar, das bis in das Mittelalter zurückreicht und das aus dieser Zeit vielerorts auch noch Bildwerke und Ausstattungsgegenstände aus vorreformatorischer Zeit bewahrt hat. Immer wieder wird in diesem Zusammenhang auf die „bewahrende Kraft des Luthertums“ hingewiesen, denn tatsächlich sind die Überlieferungsbedingungen für vorreformatorisches Kircheninventar, selbst wenn es nicht mehr weiter genutzt wurde, in den Kirchen der lutherischen Reformation weitaus günstiger gewesen als in Landschaften, in denen sich die katholische Konfession behaupten konnte. ${ }^{8}$ Von einer systematischen Beseitigung der Bilder, von einem Bildersturm ganz zu schweigen, kann nur unter dem Einfluss der Reformation Zwinglis und Calvins, nicht aber in der lutherischen Kirche die Rede sein. ${ }^{9}$ Doch selbst, wo dies im 16. Jahrhundert der Fall war, wie in Teilen der Schweiz, in der Pfalz oder im Bergischen Land, blieben vielerorts zumindest die mittelalter-

des Bistums Meißen in den päpstlichen Registern 1417-1471, in: Wolfgang Huschner/ Enno Bünz/Christian Lübke (Hg.), Italien, Mitteldeutschland, Polen. Geschichte und Kultur im europäischen Kontext vom 10. bis zum 18. Jahrhundert (Schriften zur sächsischen Geschichte und Volkskunde 42), Leipzig 2013, S. 403-530; HeIko JadATZ, Wittenberger Reformation im Leipziger Land. Dorfgemeinden im Spiegel der evangelischen Kirchenvisitationen des 16. Jahrhunderts (Herbergen der Christenheit, Sonderband 10), Leipzig 2007; Uwe SchIRMER, Unerschlossene Quellen zur Reformationsgeschichte. Kirchenrechnungen aus dem ernestinischen Kursachsen (1514-1547), in: Winfried Müller (Hg.), Perspektiven der Reformationsforschung in Sachsen. Beiträge des Ehrenkolloquiums zum 80. Geburtstag von Karlheinz Blaschke (Bausteine aus dem Institut für Sächsische Geschichte und Volkskunde 12), Dresden 2008, S. 107123.

8 Siehe die Beiträge in: Johann Michael Fritz (Hg.), Die bewahrende Kraft des Luthertums. Mittelalterliche Kunstwerke in evangelischen Kirchen, Regensburg 1997; Bettina Seyderhelm (Hg.), Goldschmiedekunst des Mittelalters. Im Gebrauch der Gemeinden über Jahrhunderte bewahrt. Eine Ausstellung der Evangelischen Kirche der Kirchenprovinz Sachsen und der Kirchlichen Stiftung Kunst- und Kulturgut in der Kirchenprovinz Sachsen im Dom zu Magdeburg, in der Stiftskirche Quedlinburg und in der Stadtkirche Wittenberg 2001 und 2002, Katalog, Dresden 2001; AnNe-Katrin ZIESAK (Hg.), Gott in Brandenburg. Christliche Lebenszeugnisse aus zwölf Jahrhunderten. Begleitbuch zur Ausstellung „Gott in Brandenburg. Zeugnisse Christlicher Kulturprägung“, 17. September 2005-8. Januar 2006, Berlin 2005. - Vgl. auch den Ausstellungskatalog KüHNE/Bünz/MüllER, Alltag und Frömmigkeit (wie Anm. 3).

9 Bob Scribner (Hg.), Bilder und Bildersturm im Spätmittelalter und in der frühen Neuzeit (Wolfenbütteler Forschungen 46), Wiesbaden 1990; Hans-Dietrich AltenDORF/Peter Jezler (Hg.), Bilderstreit. Kulturwandel in Zwinglis Reformation, Zürich 1984; Bildersturm - Wahnsinn oder Gottes Wille? Katalog der Ausstellung Bern 2000 (Vernissage 76), Heidelberg 2000; Peter Blickle/André Holenstein/Heinrich Richard Schmidt/Franz-Josef Sladeczek (Hg.), Macht und Ohnmacht der Bilder. Reformatorischer Bildersturm im Kontext der europäischen Geschichte (Historische Zeitschrift, Beihefte N. F. 33), München 2002. 
lichen Kirchenbauten erhalten und zeugen bis heute davon, dass die Grundlagen der Kirchenorganisation im Mittelalter gelegt wurden. Anstelle zahlloser regionaler und lokaler Arbeiten, die diesen Bestand präsentieren, sei auf die niederländischen kirchlichen Kunsthistoriker Regnerus Steensma (1937-2012) und Justin E. A. Kroesen verwiesen, die in mehreren attraktiv gestalteten Büchern den Reichtum erhaltener mittelalterlicher Dorfkirchen und ihres Inventars für den Bereich des lateinisch-papstchristlichen Europa dargestellt haben. ${ }^{10}$ Die Kirchen Mitteldeutschlands spielen in diesem Zusammenhang allerdings keine nennenswerte Rolle, doch bleibt zu hoffen, dass die Ausstellung „Alltag und Frömmigkeit am Vorabend der Reformation in Mitteldeutschland“ mit ihrem Katalog und der Publikation der vorbereitenden wissenschaftlichen Tagung die internationale Forschung stärker auf diesen Raum aufmerksam machen wird.

Es ist im Rahmen dieser Besprechungsmiszelle weder möglich noch sinnvoll, einen Überblick der zahlreichen Einzelpublikationen über ländliche Kirchen zu bieten. Der Versuch eines Gesamtbildes für den deutschsprachigen Raum bliebe eklektisch und müsste schön gestaltete Bildbände über die Kirchen eines bestimmten Gebietes ${ }^{11}$ ebenso erfassen wie die zahlreichen kleinen Kirchenführer, die mittlerweile von mehreren Verlagen herausgebracht und in den Kirchen angeboten werden. ${ }^{12}$ Was weithin fehlt, ist der Versuch, die Pfarrkirchen mehr oder minder größerer Landschaften bekannt zu machen, doch macht hier der mitteldeutsche Raum eine bemerkenswerte Ausnahme. Für das Gebiet der evangelischen Landeskirchen in der DDR hat nämlich die Evangelische Verlagsanstalt in Ostberlin seit den 1970er-Jahren eine ganze Reihe repräsentativer Bände herausgebracht, die vielfach in mehreren Auflagen erschienen sind. Für Sachsen, Thüringen, Sachsen-Anhalt, Brandenburg, Mecklenburg und Vorpommern sind zumeist zwei Bände über Stadtkirchen und über Dorfkirchen herausgekommen. ${ }^{13}$ Soweit sich bibliografisch ermitteln lässt, machte 1963 das Buch über die

10 Justin E. A. Kroesen/Regnerus Steensma, The interior of the medieval village church/Het middeleeuwse dorpskerkinterieur, Louvain u. a. 2004, ${ }^{2} 2012$; DiEs., Kirchen in Ostfriesland und ihre mittelalterliche Ausstattung, Petersberg 2011; Justin E. A. Kroesen, Mis! Middeleeuwse kerkinterieurs in Groningen en Ostfriesland. Uitgave naar aanleiding van de tentoonstelling in Openluchtmuseum Het Hoogeland in Warffum, 24 maart-28 mei 2012, Bedum 2012; Ders., Seitenaltäre in mittelalterlichen Kirchen. Standort - Raum - Liturgie, Regensburg 2010. - Ders./Peter Tangeberg, Die mittelalterliche Sakramentsnische auf Gotland (Schweden). Kunst und Liturgie, Petersberg 2014.

11 Weit verbreitet ist Siegrried Scharfe, Deutsche Dorfkirchen (Die Blauen Bücher), Königstein i. Ts./Leipzig 1934 (bis 1942 zahlreiche Neuauflagen); Ders., Dorfkirchen in Europa. Églises de village en Europe. Village Churches in Europe, Leipzig 1973, zugleich als Lizenzausgabe in der Reihe „Die Blauen Bücher“, Königstein i. Ts. 1973.

12 Neben den etablierten Reihen wie „Kleine Kunst- und Kirchenführer“ (Schnell \& Steiner, Regensburg), in der nach der Wiedervereinigung die Reihe „Das christliche Denkmal“ (Evangelische Verlagsanstalt, Ostberlin) aufgegangen ist, und den „DKVKunstführern“ (Deutscher Kunstverlag, Berlin) ist für unseren Raum noch hinzuweisen auf die Reihe „Der kleine sakrale Kunstführer“ (Verlag Beier \& Beran, Langenweißbach), die Günter Hummel (1952-2013) begründet hat und in der seit 2001 über 30 Hefte, Beihefte und Sonderhefte vornehmlich über Pfarrkirchen in Ostthüringen und Westsachsen erschienen sind.

13 Christian Rietschel/Bernd Langhof, Dorfkirchen in Sachsen, Berlin 1963, 81976; Heinrich Magirius/Hartmut Mai, Dorfkirchen in Sachsen, Berlin 1985, 21990; FRITZ LÖFfLER, Die Stadtkirchen in Sachsen. Mit einer geschichtlichen Einführung von Karlheinz Blaschke und einem Beitrag zur romanischen und gotischen Architektur von 
Dorfkirchen in Sachsen den Anfang, das übrigens als einziges der Reihe 1985 durch eine Neuausgabe anderer Autoren ersetzt wurde. Erst später scheint man aber bei der Evangelischen Verlagsanstalt den Plan gefasst zu haben, die einstigen Länder im Gebiet der DDR systematisch abzudecken und eine Reihe zu begründen. „Initiator der Bände war der ehemalige Verleger des Union-Verlages (CDU) Karl Wagner“, wie mir Siegfried Bräuer mitteilte; Wagner erscheint deshalb in einigen Bänden auch als Herausgeber. ${ }^{14}$ Am Anfang der Reihe „Stadt- und Dorfkirchen“ stand 1973 wieder Sachsen mit dem entsprechenden Band über die Stadtkirchen, danach kamen in schneller Folge die weiteren Bände. Nur für Sachsen-Anhalt konnte dem Buch über die Stadtkirchen keines über die Dorfkirchen an die Seite gestellt werden. ${ }^{15}$ Für Vorpommern (Landeskirche Greifswald) fehlt ein entsprechender Band über die Stadtkirchen. ${ }^{16}$ Es wäre gewiss einmal interessant, dieses kirchliche Publikationsvorhaben aus der Perspektive des Verlages und der Landeskirchen zu untersuchen. ${ }^{17}$ Dass dahinter offenbar ein Plan stand, zeigt die einheitliche Konzeption und Aufmachung der großformatigen Bände. Der Textteil besteht zumeist aus einem kirchengeschichtlichen und einem kunstgeschichtlichen Überblicksbeitrag, auf den dann ein umfangreicher Tafelteil mit durchweg sehr guten Schwarz-Weiß-Bildern und einzelnen Farbbildern folgt, die schließlich in einem weiteren Textteil („Einzeldarstellungen“) erläutert werden. Selbstverständlich wird für alle Städte und Landschaften stets nur eine Auswahl von Kirchen behandelt. Da man mit den Büchern einen breiteren Leserkreis erreichen wollte (die zahlreichen Auflagen aller Bände verdeutlichen, dass dies gelungen ist), wurde auf Einzelnachweise verzichtet. Im historischen und kunsthistorischen Überblick werden aber am Ende summarisch weiterführende Literaturhinweise geboten, u. a. auf die umfangreichen, wenn auch mittlerweile durchweg recht betagten Kunstdenkmälerinventare. Durch diese Buchreihe besteht für die Pfarrkirchen im Gebiet der Neuen Bundesländer also eine Möglichkeit des ersten thematischen Einstiegs, freilich mit dem Schwerpunkt auf der Kunst- und Ausstattungsgeschichte.

Ein Kernproblem der wissenschaftlichen Beschäftigung mit der Pfarrkirche ist, dass der Forschungsstand aus kunstgeschichtlicher Perspektive zumeist besser ist als

Heinrich Magirius, Berlin 1973, 51989; Herbert von Hintzenstern, Dorfkirchen in Thüringen, Berlin 1979, ${ }^{4}$ 1990; Klaus Mertens, Die Stadtkirchen in Thüringen, Berlin 1982, 31990; Walter May, Stadtkirchen in Sachsen/Anhalt, Berlin 1979, 21980; WolfGANG Gericke/Heinrich-Volker Schleiff/Winfried Wendland, Brandenburgische Dorfkirchen, Berlin 1975, ${ }^{4}$ 1985; ERnst BadstübneR, Stadtkirchen in der Mark Brandenburg, Berlin 1982, ${ }^{3} 1988$; Ders./Sibylle Badstübner-Gröger, Kirchen in Berlin. Von St. Nikolai bis zum Gemeindezentrum „Am Fennpfuhl“, Berlin 1987; Horst Ende, Dorfkirchen in Mecklenburg, Berlin 1975, 41985; Ders., Stadtkirchen in Mecklenburg, Berlin 1984, ${ }^{2} 1986$; Norbert Buske/Gerd Baier, Dorfkirchen in der Landeskirche Greifswald, Berlin 1984.

14 Herrn Kollegen Siegfried Bräuer (Berlin), der von 1980 bis 1991 Theologischer Direktor der Evangelischen Verlagsanstalt in Berlin war, danke ich sehr herzlich für seine ausführliche Mail vom 15. September 2014. Mehrere Bände tragen auf der Impressumsseite den Vermerk: „Dieser Band erscheint innerhalb der Reihe Stadt- und Dorfkirchen und ist herausgegeben von Karl Wagner".

15 Der Band „war zugesagt, aber der Autor lieferte nicht“, Mitteilung von Siegfried Bräuer (wie Anm. 14).

16 Dieses Thema sollte Norbert Buske bearbeiten, er „ist aber wegen vielerlei anderer Aufgaben nicht dazu gekommen“, Mitteilung von Siegfried Bräuer (wie Anm. 14).

17 Wie mir Siegfried Bräuer (wie Anm. 14) mitteilte, wird das Archiv der Evangelischen Verlagsanstalt Berlin, soweit es das Verlagsende 1989/90 überstanden hat, im Evangelischen Zentralarchiv Berlin verwahrt. 
aus historischer. Allerdings hat sich auch die Kunstgeschichte lange Zeit nicht sonderlich für den Pfarrkirchenbau interessiert ${ }^{18}$ und sich - wenn überhaupt - mit wenigen herausragenden Stadtpfarrkirchen beschäftigt, während die Masse der Kirchen der Denkmalinventarisation überlassen blieb. Die Geschichtswissenschaft und die Kirchengeschichte haben ebenfalls lange Zeit eher einen Bogen um die Thematik gemacht, sich bestenfalls um den Zusammenhang von Kirchenorganisation und Siedlungsgeschichte gekümmert, ${ }^{19}$ nur selten aber die einzelne Pfarrei in ihren lebensweltlichen Kontexten betrachtet, vermutlich, weil eine so betriebene Pfarreigeschichte als eine Spielart der Heimat- und Lokalgeschichte missverstanden wurde. ${ }^{20}$ Seitens der Amtskirche ist die Bedeutung der Pfarreigeschichte für die Identität der Gemeinde nicht immer gesehen worden, ${ }^{21}$ doch gibt es zumindest für die Sächsische Landeskirche zwei bemerkenswerte Publikationsreihen, die nicht nur mit den Bauten, sondern mit den einzelnen Pfarreien und ihrem kirchlichen Leben vertraut machen. Wer in Sachsen über Pfarrkirchen arbeitet, muss sich nämlich nicht nur auf das Inventarwerk der „Bau- und Kunstdenkmäler des Königreichs Sachsen“22 stützen, das nur für die Stadt Leipzig durch eine Neubearbeitung ersetzt worden ist, ${ }^{23}$ sondern kann auch die Beiträge in der „Sächsischen Kirchengalerie“ 24 bzw. der „Neuen Sächsischen Kirchengalerie “ 25 nutzen, mit denen im 19 . Jahrhundert seitens der evangelischen Landeskir-

18 Eine Ausnahme ist Klaus Jan Philipp, Pfarrkirchen - Funktion, Motivation, Architektur. Eine Studie am Beispiel der Pfarrkirchen der schwäbischen Reichsstädte im Spätmittelalter (Studien zur Kunst- und Kulturgeschichte 4), Marburg 1987. Vgl. auch die in Anm. 26 zitierte Arbeit von Friedrich Möbius.

19 Einige weiterführende Hinweise dazu bei EnNo Bünz, Die mittelalterliche Pfarrei in Franken. Stand, Probleme und Aufgaben der landesgeschichtlichen Atlasarbeit in Bayern, in: Konrad Ackermann/Hermann Rumschöttel (Hg.), Bayerische Landesgeschichte. Landesgeschichte in Bayern. Festgabe für Alois Schmid zum 60. Geburtstag, Teil 1 (Zeitschrift für bayerische Landesgeschichte 68, Heft 1), München 2005, S. 51-74.

20 Dazu kritisch: EnNo BüNZ, Die Pfarrei in der Stauferzeit - Romanische Stadt- und Dorfkirchen aus historischer Sicht (Vorträge im Europäischen Romanik Zentrum 3), Halle 2014 (im Druck).

21 Dieser Aspekt bedürfte gesonderter Erörterung. Anlässlich der Gründung des Vereins für die Kirchengeschichte der Provinz Sachsen erschienen dazu mehrere programmatische Aufsätze: Ausfeld, Bedeutung und Verwertung der lokalen Kirchengeschichte für die Kirchengeschichte und die allgemeine Geschichte, in: Zeitschrift des Vereins für Kirchengeschichte der Provinz Sachsen 1 (1904), S. 12-17; Büchtıng, Die lokale Kirchengeschichte in ihrer Bedeutung und Verwertung für die Gemeinde, in: ebd., S. 18-24; G. ARndt, Wert der lokalen Kirchengeschichte für den Pfarrer, in: ebd., S. 25-33.

22 Beschreibende Darstellung der älteren Bau- und Kunstdenkmäler des Königreichs Sachsen, Bd. 1-15, bearb. von Richard Steche, Bd. 16-41, bearb. von Cornelius Gurlitt, Dresden 1882-1923.

23 Heinrich Magirius u. a. (Bearb.), Die Bau- und Kunstdenkmäler von Sachsen, Stadt Leipzig: Die Sakralbauten. Mit einem Überblick über die städtebauliche Entwicklung von den Anfängen bis 1989, hrsg. vom Landesamt für Denkmalpflege Sachsen, 2 Bde., München 1995. - Die Bände behandeln nicht nur die historischen Stadtkirchen, sondern auch die zahlreichen Kirchen der seit dem 19. Jahrhundert nach Leipzig eingemeindeten Dörfer. Vgl. in diesem Zusammenhang auch das schöne Buch von Peter Findeisen/ Dirk HöHne, Die Dorfkirchen in Halle (Denkmalorte - Denkmalwerte 3), Halle 2006.

24 Sachsens Kirchen-Galerie, Bd. 1-12 und Sonderband (Die Oberlausitz), Dresden 18371845.

25 Georg Buchwald (Hg.), Neue Sächsische Kirchengalerie, Bd. 1-23, Leipzig 19001914. - Das Werk blieb wohl in Folge des Ersten Weltkrieges unvollendet. 
che der Versuch einer umfassenden historisch-statistischen Selbstbeschreibung der kirchlichen Verhältnisse unternommen wurde. Für die „Neue Sächsische Kirchengalerie“ zeichnete der Kirchenhistoriker und Lutherforscher Georg Buchwald verantwortlich, der für die einzelnen Kirchspiele allerdings auf die mehr oder minder ausgereiften Darstellungen der Ortsgeistlichen angewiesen war. Für Sachsen gibt es auch eine mehr als hundert Jahre alte Gesamtdarstellung der Bauformen und Ausstattung der Dorfkirchen aus der Feder eines Architekten, die als Gesamtentwurf und aufgrund des reichen Bildmaterials durchaus noch von Wert ist, historisch aber überhaupt nicht befriedigen kann. ${ }^{26}$ Die Pfarrei besteht eben nicht nur aus einem Kirchenbau und seiner Ausstattung, sondern sie ist eine geistliche Institution mit vielfältigen herrschaftlichen, rechtlichen, sozialen, ökonomischen und kulturellen Bezügen. Die kunst- und baugeschichtliche Untersuchung müsste deshalb um eine historisch-statistische Darstellung erweitert werden, wie sie im Rahmen des Großvorhabens "Germania Sacra“ für die Pfarrorganisation des Bistums Bamberg mustergültig vorgelegt worden ist. Gegliedert nach den größeren kirchlichen Organisationseinheiten der Archidiakonate werden die Pfarreien mit ihren Filialkirchen und -kapellen nach einem einheitlichen Schema beschrieben. Nach Ortsname und Patrozinium ${ }^{27}$ wird die erste Erwähnung der Kirche (bzw. des Geistlichen) angegeben, dann werden die Patronatsverhältnisse dargestellt und schließlich der Umfang des Kirchspiels beschrieben. Sofern in der Pfarrkirche Nebenaltäre mit Vikarien oder Kapellen bestanden, schließt sich deren Darstellung an. ${ }^{28}$ Dass Sachsen durch diesen Band zumindest am Rande berührt wird, weil einige Pfarreien und Filialkirchen im Nordosten des Bistums auf sächsischem

26 Otto Gruner, Die Dorfkirche im Königreich Sachsen. Eine Darstellung ihrer Entstehung, Entwicklung und baulichen Eigenart, Leipzig 1904. Der Verfasser möchte ein Interesse für die alten Dorfkirchen wecken, die noch „die Verkörperung des Gemütsund Glaubenslebens ihrer Gemeinden" waren (S. 5). Diese wie andere Formulierungen legen den Schluss nahe, dass der Verfasser der „Deutschen Dorfkirchenbewegung“ nahestand, als deren Organ sei 1907 die Zeitschrift „Die Dorfkirche. Illustrierte Monatsschrift zur Pflege des religiösen Lebens in heimatlicher und volkstümlicher Gestalt" erschien. Vgl. dazu Angela Treiber, Volkskunde und evangelische Theologie. Die Dorfkirchenbewegung 1907-1945, Köln/Weimar/Wien 2004. - Regional weiter ausgreifende Arbeiten über Dorfkirchen in Sachsen sind seitdem nur vereinzelt entstanden. Die Dissertation von Wilhelm Liebrich, Wehrhafte Kirchen in Sachsen, Hauptband und Anlagenband, Diss. (masch.) Dresden 1926, blieb ungedruckt. Wichtiger ist das Buch von Klaus Mertens, Romanische Saalbauten innerhalb der mittelalterlichen Grenzen des Bistums Meißen (Studien zur katholischen Bistums- und Klostergeschichte 14), Leipzig 1967. - Weitere Literaturnachweise bei FRIEDRICH Мӧвгus, Die Dorfkirche im Zeitalter der Kathedrale (13. Jh.). Plädoyer für eine strukturgeschichtliche Vertiefung des Stilbegriffs (Sitzungsberichte der Sächsischen Akademie der Wissenschaften zu Leipzig. Philologisch-Historische Klasse 128, H. 3), Berlin 1988. Seitdem ist erschienen: Claudia Trummer, Früher Backsteinbau in Sachsen und Südbrandenburg (Kultur- und Lebensformen in Mittelalter und Neuzeit 4), Schöneiche b. Berlin 2011.

27 Für die sächsischen Kirchen ist hier noch immer die Dissertation von Herbert Helbig, Untersuchungen über die Kirchenpatrozinien in Sachsen auf siedlungsgeschichtlicher Grundlage (Historische Studien 361), Berlin 1940, eine grundlegende Vorarbeit.

28 Erich Freiherr von Guttenberg/Alfred Wendehorst, Das Bistum Bamberg, Teil 2: Die Pfarreiorganisation (Germania Sacra. Zweite Abteilung: Die Bistümer der Kirchenprovinz Mainz, Bd. 1: Das Bistum Bamberg), Berlin 1966, das Gliederungsschema hier S. 44 beschrieben. 
Boden lagen, sei erwähnt. ${ }^{29}$ Nach dem neuen Konzept des Projekts der „Germania Sacra“, das seit 2008 als Langfristprojekt bei der Akademie der Wissenschaften zu Göttingen betrieben wird, spielt die detaillierte Aufarbeitung der Pfarreiorganisation allerdings keine Rolle mehr. ${ }^{30}$ So wird es künftig nicht nur in Sachsen Aufgabe der landesgeschichtlichen Forschung sein, entsprechende Darstellungen zu schaffen, wobei es sinnvoll sein dürfte, über das recht enge Bearbeitungsschema des genannten Germania-Sacra-Bandes hinauszukommen und ein komplexeres Beschreibungsmodell für die Pfarreien zu konzipieren. ${ }^{31}$

Nach der Friedlichen Revolution und der deutschen Wiedervereinigung haben sich auch für die Erforschung der Dorfkirchen in den neuen Bundesländern neue Perspektiven eröffnet. Vor allem Umbau- und Restaurierungsmaßnahmen ermöglichten der Archäologie und Bauforschung viele neue Untersuchungsmöglichkeiten. In den Braunkohletagebaugebieten Brandenburgs (Niederlausitz), Sachsens und SachsenAnhalts konnten nun ganze Dörfer mitsamt der Kirche archäologisch untersucht werden. ${ }^{32}$ Es war deshalb kein Zufall, dass sich vor allem die Landesämter für Archäologie der Thematik angenommen und der Erforschung von Dorfkirchen durch Tagungen weitere Impulse gegeben haben. Bereits 1992 hat das Landesmuseum für Vorgeschichte in Dresden eine Tagung zur Archäologie und Baugeschichte früher Kirchen in Sachsen

29 Vgl. ebd., Karte 5 und Blaschke/Ковuch, Kirchenorganisation (wie Anm. 5), S. 49 f.

30 Jasmin Höven/Bärbel Kröger/Nathalie Kruppa/Christian Popp, Neuausrichtung der Germania Sacra an der Akademie der Wissenschaften zu Göttingen, in: Blätter für deutsche Landesgeschichte 143 (2007), S. 231-241.

31 Ein differenziertes Beschreibungsschema bietet: Ludwig Anton Doll (Hg.), Palatia Sacra. Kirchen- und Pfründebeschreibung der Pfalz in vorreformatorischer Zeit, hrsg. auf Grund der Vorarbeiten von Franz Xaver Glasschröder, Teil I: Das Bistum Speyer. Der Archidiakonat des Dompropstes von Speyer, Bd. 1-5, Mainz 1988-2009. Vgl. dazu Ludwig Anton Doll, Plan und Verwirklichung einer Palatia Sacra - Gedanken zum "Nachlaß Glasschröder“, in: Arbeitsgemeinschaft für geschichtliche Landeskunde am Oberrhein e. V., Karlsruhe, 263. Protokoll (Arbeitssitzung vom 14. November 1986), S. 1-18 (mit Diskussion S. 18-29); Volker RöDEL, Die Pfarrorganisation im linksrheinischen Teil des Bistums Speyer im Mittelalter. Ein Überblick, in: Archiv für mittelrheinische Kirchengeschichte 44 (1992), S. 13-40, hier S. 13-15 und EnNo BüNZ, „Des Pfarrers Untertanen“? Die Bauern und ihre Kirche im späten Mittelalter, in: KuRT Andermann/Oliver Auge (Hg.), Dorf und Gemeinde. Grundstrukturen der ländlichen Gesellschaft in Spätmittelalter und Frühneuzeit (Kraichtaler Kolloquien 8), Epfendorf 2012, S. 153-191, hier S. 166-168.

32 Am umfangreichsten dokumentiert ist die Ausgrabung des Pfarrdorfes Breunsdorf südlich von Leipzig, siehe hier: Judith Oexle (Hg.), Kirche und Friedhof von Breunsdorf. Beiträge zu Sakralarchitektur und Totenbrauchtum in einer ländlichen Siedlung. Mit Beiträgen von Hans-Joachim Gregor u. a. (Veröffentlichungen des Landesamtes für Archäologie mit Landesmuseum für Vorgeschichte 35), Dresden 2002. Gut dokumentiert ist auch das abgebaggerte Kirchdorf Horno in der Niederlausitz; vgl. zur Kirche Yngve Jan Holland/Andreas PotThoff, Die Dorfkirche St. Nikolaus, in: Detlef Karg/Franz Schopper (Hg.), Horno - Zur Kulturgeschichte eines Niederlausitzer Dorfes, Bd. 2: Historische Bauforschung, Historische Geographie, Botanik, Sprachwissenschaft, Wünsdorf 2007, S. 299-313. Ein Beitrag zur Geschichte der Pfarrei fehlt. Thomas Rudert, Soziale Struktur und kulturelle Praxis - Untersuchungen zur Geschichte von Horno nach der archivalischen Überlieferung bis 1850, in: Horno (s. o.), Bd. 1: Geschichte, Ethnographie, Wünsdorf 2006, S. 19-320, geht S. 23-25 nur auf die Nennung der Pfarrei in der Meißener Bistumsmatrikel von 1495 ein. 
durchgeführt, die zwei Jahre später publiziert wurde. ${ }^{33}$ Der Schwerpunkt lag dabei auf romanischen Kirchenbauten. Das Brandenburgische Landesamt für Denkmalpflege hat 2006 gemeinsam mit weiteren Institutionen in Luckenwalde ebenfalls eine Tagung über frühe Kirchen im Blickfeld von Archäologie und Bauforschung veranstaltet, deren Beiträge bereits ein Jahr später gedruckt werden konnten. ${ }^{34}$ Untersuchungen an Dorfkirchen standen dabei im Mittelpunkt, doch wurden auch Dom-, Stadt- und Klosterkirchen behandelt. Zumindest ansatzweise versuchte man auch, landesgeschichtliche Aspekte mit einzubeziehen. ${ }^{35}$ Das Thüringische Landesamt für Denkmalpflege und Archäologie hat 2009 in Weimar gemeinsam mit der Archäologischen Gesellschaft in Thüringen ein Kolloquium über archäologische und bauhistorische Untersuchungen an und in Kirchen Thüringens veranstaltet, deren Ergebnisse seit 2014 in einem Sammelband greifbar sind. ${ }^{36}$ Auch dort waren Historiker und Kirchenhistoriker in die Tagung einbezogen. ${ }^{37}$ Während auf diesen Kolloquien neben übergreifenden Themen z. T. auch Stadt-, Kloster- und Wallfahrtskirchen behandelt wurden, widmete sich bereits 2001 eine Veranstaltung des Instituts für Kunstgeschichte der Martin-Luther-Universität Halle-Wittenberg ganz der mittelalterlichen Dorfkirche in den Neuen Bundesländern, wobei neben Forschungsstand und -aufgaben auch die vielfach offene Nutzungsproblematik erörtert wurde, ein anhaltend drängendes und aktuelles Problem. ${ }^{38}$ Eine Folgetagung, die sich auf Aspekte der Wehrhaftigkeit mittel-

33 Judith Oexle (Hg.), Frühe Kirchen in Sachsen. Ergebnisse archäologischer und baugeschichtlicher Untersuchungen (Veröffentlichungen des Landesamtes für Archäologie mit Landesmuseum für Vorgeschichte 23), Stuttgart 1994.

34 Kirchen des Mittelalters in Brandenburg und Berlin. Archäologie und Bauforschung (Denkmalpflege in Berlin und Brandenburg 3), Petersberg 2007.

35 Z. B. durch die Beiträge von Heinz-Dieter Heimann, Frühe Kirchen - Gebaute Zeugen. Organisations- und Deutungsmerkmale der Kirchengebäude und mittelalterlichen Kirchenlandschaft in der Mark Brandenburg im Widerstreit des aktuellen Stadtumbaus, in: Kirchen des Mittelalters in Brandenburg und Berlin (wie Anm. 34), S. 8-20; Matthias Friske, Kirchen und ihre Bedeutung für die Landesgeschichte, in: ebd., S. 21-25 (vor allem Bezug zum Landesausbau); UlRICH WaAcK, Dorfkirchenbau und Ökonomie. Über den Zusammenhang der baulichen Gestalt mittelalterlicher Dorfkirchen auf dem Barnim mit Siedlungsmerkmalen, in: ebd., S. 26-37; siehe dazu auch unten Anm. 59.

36 Mittelalterliche Kirchen in Thüringen. Beiträge der Tagung „Archäologische und bauhistorische Untersuchungen an und in Kirchen Thüringens“, Weimar, 16./17. März 2009, ausgerichtet vom Thüringischen Landesamt für Denkmalpflege und Archäologie und der Archäologischen Gesellschaft in Thüringen (Alt-Thüringen 43 [2012/13] = Arbeitshefte des Thüringischen Landesamtes für Denkmalpflege und Archäologie. Archäologische Reihe 43), Langenweißbach 2014.

37 Helge Wittmann, $\mathrm{Zu}$ den Anfängen des Niederkirchenwesens in Thüringen, in: Mittelalterliche Kirchen in Thüringen (wie Anm. 36), S. 21-31; Enno Bünz, Pfarreiorganisation, Kirchenbauten und -ausstattung im spätmittelalterlichen Thüringen. Historische Aspekte archäologischer und bauhistorischer Untersuchungen, in: ebd., S. 53-72; ERnst Koch, Die Bedeutung der Pfarrkirche für die mittelalterliche Dorfgesellschaft, in: ebd., S. 317-340.

38 Wolfgang Schenkluhn (Hg.), Die mittelalterliche Dorfkirche in den Neuen Bundesländern I. Forschungsstand - Forschungsperspektiven - Nutzungsprobleme (Hallesche Beiträge zur Kunstgeschichte 3), Halle 2001. 
alterlicher Dorfkirchen konzentrierte, wurde 2005 veranstaltet. ${ }^{39}$ Beide Tagungen verzichteten auf die Einbeziehung von Landes- und Kirchenhistorikern.

Seit 2001 erscheint im Berliner Lukas Verlag die Buchreihe „Kirchen im ländlichen Raum“, die von Bernhard Janowski und Dirk Schumann herausgegeben wird und mittlerweile fünf Monografien und Sammelbände umfasst. ${ }^{40}$ Band 6 über die Dorfkirchen der Niederlausitz wurde bereits in dieser Zeitschrift besprochen, ${ }^{41}$ doch war schon seit längerem beabsichtigt, die Reihe insgesamt zu würdigen, für die weitere Bände bereits in Vorbereitung sind. ${ }^{42}$ Dabei mag es Zufall sein, dass ausgerechnet das Land Brandenburg im Mittelpunkt dieser Buchreihe steht, die jedenfalls von der Titelgebung her nicht regional eingeschränkt ist. Das hängt zum einen gewiss mit dem Verlag zusammen, der sich mit seinem kunst- und regionalgeschichtlichen Buchprogramm in Brandenburg bestens etabliert hat, Bau- und Kunsthistoriker wie Matthias Friske und Dirk Schumann zu seinen Autoren zählt, die sich besonders intensiv um die mittelalterlichen Kirchenbauten im Land kümmern, und ein anhaltendes Interesse an den alten Dorfkirchen im Lande besteht, das vom Förderkreis Alte Kirchen BerlinBrandenburg e. V. tatkräftig unterstützt wird. ${ }^{43}$ Weiter mag auch eine Rolle spielen, dass die Zahl der romanischen Dorfkirchen in Brandenburg besonders hoch ist und in Folge des hochmittelalterlichen Siedlungsganges in fast jedem Dorf eine Kirche existiert; entsprechend sind die Kirchenbauten - ganz im Sinne der eingangs zitierten Äußerung Theodor Fontanes - Denkmäler der lokalen Identität, auch wenn sie angesichts fortschreitender Entkirchlichung und Ausdünnung von Seelsorgestrukturen nicht allenthalben mehr ihrem eigentlichen Zweck dienen.

Kommen wir nun zu den bisher erschienenen fünf Bänden der Buchreihe, darunter drei Monografien. Matthias Friske ist evangelischer Pfarrer und studierte an der Humboldt-Universität Berlin neben Theologie auch Geschichte und Kunstgeschichte. Mit der umfangreichen Monografie über die mittelalterlichen Kirchen auf dem Barnim (die Landschaft nordöstlich des Urstromtals der Spree) wurde er 2000 von dem Landeshistoriker Winfried Schich promoviert. ${ }^{44}$ Eine weitere Monografie hat Friske 2007 über die mittelalterlichen Kirchen im westlichen Fläming und Vorfläming vorgelegt. ${ }^{45}$ Das angekündigte Buch über die mittelalterlichen Kirchen in der Uckermark dürfte dem gleichen Ansatz folgen und lässt erwarten, dass der Verfasser nach und nach die vor-

39 Dirk Höhne/Christine Kratzke (Hg.), Die mittelalterliche Dorfkirche in den Neuen Bundesländern II. Form - Funktion - Bedeutung (Hallesche Beiträge zur Kunstgeschichte 8), Halle 2006.

40 Der angekündigte Band 2 der Reihe wird wohl nicht mehr erscheinen: BERnd JANOwsKI (Hg.), Wie viele Kirchen braucht das Land?

41 Anne Gehrmann/Dirk Schumann (Hg.), Dorfkirchen in der Niederlausitz. Geschichte - Architektur - Denkmalpflege (Kirchen im ländlichen Raum 6), Berlin 2011, Rezension in: NASG 83 (2012), S. 334-336.

42 Angekündigt sind: MATTHIAs FrisKe, Die mittelalterlichen Kirchen in der nördlichen und östlichen Uckermark. Geschichte - Architektur - Ausstattung (Kirchen im ländlichen Raum 7), Berlin 2014; Wolf-Dietrich Meyer-Rath/Friedrich von Klitzing (Hg.), Die spätmittelalterlichen Wandmalereien in der Dorfkirche zu Demerthin (Kirchen im ländlichen Raum 8), Berlin 2014.

43 Vgl. die Homepage http://www.altekirchen.de/ hrsg. vom Förderkreis Alte Kirchen Berlin-Brandenburg e. V. [Zugriff am 12. September 2014].

44 Matthias Friske, Die mittelalterlichen Kirchen auf dem Barnim. Geschichte - Architektur - Ausstattung (Kirchen im ländlichen Raum 1), Berlin 2001.

45 Ders., Mittelalterliche Kirchen im westlichen Fläming und Vorfläming (Kirchen im ländlichen Raum 5), Berlin 2007. 
reformatorischen Kirchen des Landes Brandenburg aufarbeiten wird. Für Berlin ist dies bereits in anderem Zusammenhang geschehen. ${ }^{46}$ Der Aufbau der beiden Monografien über den Barnim und den Fläming ist weitgehend identisch. Beide Untersuchungsgebiete gehörten zum mittelalterlichen Bistum Brandenburg, doch spielen Fragen der Diözesanzugehörigkeit und der pfarreiübergreifenden kirchlichen Organisation in den beiden Arbeiten keine besondere Rolle. Auf einen kurzen geschichtlichen Überblick folgt die Darstellung der Kirchen nach Orten. Auf dem Barnim sind es 133 erhaltene mittelalterliche Kirchen (mehrere Gotteshäuser, z. T. auch Klöster, gab es in den Städten des Untersuchungsgebietes, also in Altfriedland, Altlandsberg, Bernau, Eberswalde, Freienwalde, Strausberg und Wriezen), auf dem Fläming ungefähr 40, wobei dort die Stadt Zerbst mit ihren zahlreichen Pfarr- und Klosterkirchen eine besondere Stellung einnimmt. Die Ortsartikel sind einheitlich aufgebaut, nennen die wichtigste Literatur, bieten einige historische Informationen (Ersterwähnung, Pfarrhufen, Patronat, insgesamt aber eher eklektisch), beschreiben das Äußere und das Innere des Kirchenbaus (mit Nennung der älteren Ausstattung, z. T. auch unter Rückgriff auf Inventare u. a. Quellen ${ }^{47}$ und schließen mit einer resümierenden Einordnung. Die Kirchenbeschreibungen bilden die Grundlage der Auswertung, die am Ende dieser Monografien steht, sowohl Bauteile als auch Ausstattungsstücke würdigt, für die Datierung auch neue dendrochronologische Daten berücksichtigt und - systematischer im Band über die Kirchen des Flämings - auf historische Aspekte wie Flurgrößen (Zusammenhang mit den Dimensionen des Kirchenbaus), Finanzierung, Filiationsverhältnisse und Baukosten eingeht. Wichtig ist der Nachweis, dass die Dorfkirchen der Kolonisationszeit (im Zerbster Raum ab 1150, auf dem Barnim ab 1230) zunächst aus Holz gebaut und erst in einer zweiten Phase im Laufe des 13. Jahrhunderts zumeist durch Feldsteinbauten ersetzt wurden. ${ }^{48}$ Die Befunde können hier im Einzelnen

46 Berlin und seine Bauten, Teil 6: Sakralbauten. Mit Beiträgen von Marcus Cante u. a., Berlin u. a. 1997. Hier sind die Ergebnisse der ungedruckten Untersuchung von Markus Cante, Mittelalterliche Dorfkirchen in Berlin, 2 Teilbde., FU Berlin Magisterarbeit (masch.) 1987, eingeflossen.

47 Zur Ausstattung brandenburgischer Stadt- und Dorfkirchen sind mittlerweile mehrere weiterführende Werke erschienen: Mittelalterliche Wandmalerei in Brandenburg, Bd. 1: Der Südosten - die Brandenburgische Lausitz (Forschungen und Beiträge zur Denkmalpflege im Land Brandenburg 11), Worms 2010; Ute Bednarz u. a. (Hg.), Die mittelalterlichen Glasmalereien in Berlin und Brandenburg. Mit einer kunstgeschichtlichen Einleitung von Peter Knüvener, 2 Bde. (Corpus Vitrearum Medii Aevi 22), Berlin 2010; Peter Knüvener, Die spätmittelalterliche Skulptur und Malerei in der Mark Brandenburg (Forschungen und Beiträge zur Denkmalpflege im Land Brandenburg 14), Worms 2011; Franziska Nentwig (Hg.)/Peter Knüvener (Bearb.), Mittelalterliche Kunst aus Berlin und Brandenburg im Stadtmuseum Berlin, Berlin 2011.

48 Friske, Fläming (wie Anm. 45), S. 157 f.; Friske, Barnim (wie Anm. 44), S. 389, Anm. 3 bemüht als Vergleichsbeispiel für den Übergang vom Holz- zum Steinbau den romanischen Lübecker Dom und verweist auf die Mitteilung bei Arnold von Lübeck I,

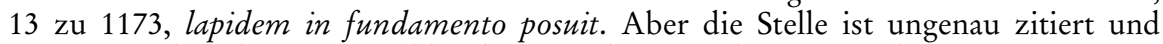
missverstanden, denn in Arnolds Chronica Slavorum, hrsg. von Johannes Martin Lappenberg (Monumenta Germaniae Historica. Scriptores rerum Germanicarum in usum scholarum [14]), Hannover 1868, S. 35, heißt es, der Herzog habe zusammen mit dem Bischof primum [...] lapidem in fundamento gelegt. Es geht hier um den rituellen Akt der Grundsteinlegung, der nichts über die Bauweise aussagt, vgl. dazu ENNo BüNZ, „lapis angularis“ - die Grundsteinlegung 1010 als Schlüssel für den mittelalterlichen Kirchenbau von St. Michael in Hildesheim, in: Gerhard Lutz/Angela Weyer (Hg.), 1000 Jahre St. Michael in Hildesheim. Kirche - Kloster - Stifter (Schriften des Hornemann 
natürlich nicht referiert werden und bedürfen auch vielfach noch einer präziseren historischen Einordnung. Das Interesse richtet sich vor allem auf die romanischen Kirchenbauten. Auch wenn der Autor in beiden Büchern über die Reformation hinaus bis ins 17. Jahrhundert ausgreift, werden die spätmittelalterlichen Jahrhunderte nur knapp behandelt. Auffällig ist z. B. in den Kirchen des Barnim die geringe Zahl von Baumaßnahmen der Spätgotik, ${ }^{49}$ ebenso die geringe Zahl von gestifteten Messpriesterstellen (Vikarien) mit Nebenaltären, doch bleibt hier unklar, welchen Zusammenhang der Verfasser mit den Kommunikantenzahlen der Dörfer sieht, auf die er diesbezüglich verweist. ${ }^{50}$ Die Vikarien waren nicht mit Seelsorgeaufgaben verbunden, und deshalb ist ihre Anzahl nicht von der Bevölkerungsgröße abhängig. Bei der Behandlung der erhaltenen Ausstattungsstücke wird immer wieder deutlich, wie wichtig es ist, ergänzend die Quellen des 16. Jahrhunderts heranzuziehen, die beispielsweise Orgeln nachweisen, ${ }^{51}$ die sich aus vorreformatorischer Zeit aber nirgends erhalten haben. Der Um- und Ausbau der Pfarrkirchen im 15. und frühen 16. Jahrhundert wird im Vergleich zur Gründungszeit des 13. Jahrhunderts eher kursorisch behandelt, 52 obwohl sich hier gewiss noch am ehesten Schriftquellen fänden, aber hier liegt offenkundig nicht der Interessenschwerpunkt des Verfassers. ${ }^{53}$ Ein weiteres Problem liegt darin, dass sich Friske zu wenig die fundamentale Bedeutung des mittelalterlichen Kirchenrechts vergegenwärtigt. Es mag sein, dass sich die Filiationsverhältnisse nicht immer sicher rekonstruieren lassen, aber dass „die Verbindungen zwischen den Kirchen, ebenso wie in der Neuzeit, durchaus flexibel gewesen sein dürften ", 54 ist schwer zu glauben; sowohl die Pfarrer als auch die Gemeinden werden ein klares Bild vom Umfang des Kirchspiels gehabt haben. Von großer Relevanz ist auch die Vermögensorganisation der Pfarrkirchen, vor allem die Unterscheidung von Pfarrpfründe und Kirchenstiftung (Fabrikgut) sowie die Finanzierung des Pfarrkirchenbaus. ${ }^{55}$ Die vielfach adligen Patronatsherren, also Kirchenstifter, werden einen erheblichen Teil der Finanzierung getragen haben, wie es im Corpus iuris canonici in den Bestimmungen über das Patronatsrecht vorgesehen war, denn aus dieser Leistung resultierte überhaupt erst das ,ius patronatus'. ${ }^{56}$ Darüber hinaus werden sich die Pfarrgemeinden mit Spenden

Instituts 14), Petersberg 2012, S. 77-87. Bedenken gegen die Quellenbenutzung Friskes hatte bereits Dietrich Kurze in seiner Rezension in: Jahrbuch für Berlin-Brandenburgische Kirchengeschichte 64 (2003), S. 333-335 anhand weiterer Beispiele geäußert.

Friske, Barnim (wie Anm. 44), S. 422-425.

50 Ebd., S. 434 f.

51 Ebd., S. 465.

52 Ebd., S. 496-498.

53 Die Dissertation wurde 2000 abgeschlossen, sodass der Verfasser den fundamentalen Handbuchbeitrag von Dietrich Kurze, Das Mittelalter. Anfänge und Ausbau der christlichen Kirche in der Mark Brandenburg (bis 1535), in: Gerd Heinrich (Hg.), Tausend Jahre Kirche in Berlin-Brandenburg, Berlin 1999, S. 15-146 noch hätte heranziehen können. Der Beitrag nun wiederabgedruckt in: Ders., Berlin-Brandenburgische Kirchengeschichte im Mittelalter. Neun ausgewählte Beiträge, hrsg. von Marie Luise Heckmann u. a. (Bibliothek der Brandenburgischen und Preussischen Geschichte 9), Berlin 2002, S. 1-110.

54 Friske, Fläming (wie Anm. 45), S. 124 f.

55 Ebd., S. 126-128 über die Baukosten.

56 Vgl. Peter Landau, Ius patronatus. Studien zur Entwicklung des Patronats im Dekretalenrecht und der Kanonistik des 12. und 13. Jahrhunderts (Forschungen zur kirchlichen Rechtsgeschichte und zum Kirchenrecht 12), Köln/Wien 1975, zusammenfassend Ders., Artikel: Patronat, in: Theologische Realenzyklopädie, Bd. 26, Berlin 1996, S. 106-108. 
und Stiftungen auf dem Weg über die Kirchenfabriken beteiligt haben, an deren Existenz - ob freilich flächendeckend, sei dahingestellt - schon im 13. Jahrhundert im Bistum Brandenburg nicht zu zweifeln ist. ${ }^{57}$ Auch hier bleibt in der Darstellung Friskes vieles unklar und vage. Welche Rolle Ablässe bei der Finanzierung des Kirchenbaus (und -unterhalts) im späten Mittelalter spielten, wird gar nicht angesprochen, obwohl dafür sogar die Registerüberlieferung der Römischen Kurie aussagekräftig sein kann. ${ }^{58}$

Diese Monita übersehen nicht, dass die Quellenlage für die Geschichte des Niederkirchenwesens im Bistum Brandenburg insgesamt eher dürftig ist und sich gerade die ökonomische Seite des kirchlichen Lebens (Pfarrerfinanzierung und Kirchenbau) vielfach nur unter Ausgriff auf die reicheren Quellen der Frühen Neuzeit werden klären lassen. Einen originellen methodischen Weg hat Ulrich Waack beschritten, der sich als Oberregierungsrat a. D. im Ruhestand dem Geschichtsstudium an der HumboldtUniversität zugewandt und 2004 mit einer Magisterarbeit über Kirchenbau und Ökonomie abgeschlossen hat. Die Arbeit ist 2009 in überarbeiteter Form in der Reihe „Kirchen im ländlichen Raum“ erschienen.59 Man ist einerseits beeindruckt von der detaillierten Materialfülle, die der Verfasser über die romanischen Kirchen des Barnim, insbesondere ihre Grundrissdisposition und Turmgestaltung, ausbreitet, auf der anderen Seite aber auch irritiert von einer gewissen "Geschichtsferne“ dieser Untersuchung; denn angesichts des bereits erwähnten Quellenmangels lässt sich der Autor nicht auf Urkunden und Akten ein, erörtert auch nicht Patronatsrechte und Ausstattungsverhältnisse von Pfarrpfründe und Kirchenstiftung, über die sich in den Schriftquellen doch manches fände, sondern widmet sich quantitativ-qualitativen Analysen des (angenommenen) Verhältnisses von Kirchenbau und Bodengüte. Der Verfasser möchte - kurz und bündig gesagt - nachweisen, dass die Dorfgemeinden mit den ertragsfähigsten Böden die größten und aufwendigsten Kirchen gebaut haben, und dass diese Kirchen zumeist auch die ältesten Gotteshäuser sind. Wie detailliert sich Ulrich Waack in die Bauformen der Dorfkirchen und die lokalen Verhältnisse eingearbeitet hat, ist schon daran ablesbar, dass er die baulichen Merkmale mit bis zu 57 Kriterien beschreibt und die Kirchenbauten $\mathrm{zu}$ „Kostenaufwandsgruppen“ zusammenfasst, während die Siedlungen bzw. ihre Gemarkungen anhand von maximal 27 Kriterien beschrieben werden. ${ }^{60}$ Ein solcher methodischer Ansatz entzieht sich der historischen Kritik, da für die Auftragsvergabe, Gestaltungsvorgaben und Baufinanzierung der

57 Das belegen mehrere Formulare bei Franz WinTER, Ein Formelbuch des dreizehnten Jahrhunderts aus der Magdeburger Kirchenprovinz, in: Geschichtsblätter für Stadt und Land Magdeburg 12 (1877), S. 1-40.

58 Für den Bereich des Bistums Meißen, zu dem auch die Niederlausitz gehörte, habe ich aus den römischen Registern von 1417 bis 1471 sämtliche Belege zusammengestellt, darunter auch etliche Belege von päpstlichen Ablässen für Dorfkirchen: BüNZ, Römische Kurie (wie Anm. 7), S. 510-514 und die dort beiliegenden Karten. Vgl. nun auch den Katalogband Martin Sünder/Helge Wittmann (Red.), Frömmigkeit in Schrift und Bild. Illuminierte Sammelindulgenzen im mittelalterlichen Mühlhausen (Ausstellungen des Stadtarchivs Mühlhausen 3 = Schriftenreihe der FriedrichChristian-Lesser-Stiftung 29), Petersberg 2014.

59 UlRICH WAACK, Kirchenbau und Ökonomie. Zur Beziehung von baulichen Merkmalen mittelalterlicher Dorfkirchen auf dem Barnim und dessen Wirtschafts- und Siedlungsgeschichte (Kirchen im ländlichen Raum 4), Berlin 2009. - Vgl. auch Ders., Dorfkirchenbau und Ökonomie. Über den Zusammenhang der baulichen Gestalt mittelalterlicher Dorfkirchen auf dem Barnim mit Siedlungsmerkmalen, in: Kirchen des Mittelalters (wie Anm. 34), S. 26-37.

60 Vgl. die beiden Tabellen der Merkmale bei Ders., Kirchenbau und Ökonomie (wie Anm. 59), S. 28 und 56. 
Pfarrkirchen im 13. Jahrhundert keine Quellen zur Verfügung stehen, ja selbst über die Baumeister bzw. Bauhütten im ländlichen Bereich nichts bekannt ist. Sofern der Verfasser aus der Korrelation von Baugestalt und Dorfgemarkung Rückschlüsse auf die Datierung der Kirchenbauten zieht, bleibt abzuwarten, wie sich dazu die Ergebnisse der Bauforschung und Archäologie verhalten. Dabei ist unbestritten, dass der „ökonomische Faktor im Dorfkirchenbau“ (S. 129) eine Rolle gespielt haben muss. Zu den offenen Fragen, die der Verfasser abschließend aufwirft, gehören der überregionale Vergleich, die Frage der Ertragsfähigkeit mittelalterlicher Böden und der Zusammenhang von Finanzierung und Bauherr. ${ }^{61}$ Hier wird deutlich, dass der Verfasser bei dieser Frage durchaus besser als Matthias Friske zu differenzieren vermag. So wird beispielsweise das Problem der geteilten Kirchenbaulast mit „Mischfinanzierungen“ angesprochen. ${ }^{62}$ Gängige Finanzierungswege wie Ablässe zugunsten des Kirchenbaus werden nur beiläufig thematisiert. Hier ist $\mathrm{zu}$ fragen, ob es bischöfliche oder kuriale Ablassurkunden für Dorfkirchen im Bistum Brandenburg gibt? Andere Möglichkeiten, Geld für den Kirchenbau zu sammeln, wie beispielsweise die im späten Mittelalter sehr verbreiteten Petitorien, werden hingegen gar nicht bedacht. ${ }^{63}$ Gerne wüsste man auch, ob und seit wann Kirchenrechnungen für die Dorfkirchen im Bistum Brandenburg und insbesondere auf dem Barnim überliefert sind. ${ }^{64}$ Selbstverständlich reichen solche Rechnungen auch in anderen Landschaften nicht in das 13. Jahrhundert zurück, also in die Hauptentstehungsphase der brandenburgischen Dorfkirchen, aber sie dokumentieren doch Baumaßnahmen sowie Anschaffungen von Inventarstücken oder der Reparatur im 15. oder 16. Jahrhundert. ${ }^{65}$

Werfen wir abschließend noch einen Blick in die beiden Sammelbände der Reihe „Kirchen im ländlichen Raum“. Der umfangreiche Band über Dorfkirchen mit Beiträgen zu Architektur, Ausstattung und Denkmalpflege ist aus einer Tagung hervorgegangen, die 2003 in Prenzlau stattfand. ${ }^{66}$ Die Publikation reiht sich also ein in die bereits erwähnten Tagungsvorhaben in Sachsen, Sachsen-Anhalt und Brandenburg, ${ }^{67}$ ist thematisch aber stärker auf Fragen der Kunst- und Baugeschichte von Dorfkirchen in Brandenburg konzentriert. Die 23 Beiträge sind fünf Themenkomplexen zugeordnet, von denen der erste, mit „Geschichte“ überschriebene, historisch enttäuschend ist, denn es geht um Siedlungsarchäologie (Eike Gringmuth-Dallmer) und um Kirchenarchäologie (Markus Agthe), nicht aber um Fragestellungen, die der Historiker aufgrund schriftlicher Quellen untersucht. Tatsächlich enthält der Band keinen einzigen landes- oder kirchengeschichtlichen Beitrag. Dafür ist der Themenbereich „Architektur“ breit angelegt. Es geht um Baumaterialien wie Feldstein (Ernst Badstübner) und

61 Ebd., S. 142-144.

62 Ebd., S. 144 f.

63 Dazu für Thüringen BüNz, Die Bauern und ihre Kirche (wie Anm. 7), S. 243-245.

64 Die Kirchenrechnungen bis 1600 werden z. B. in den Regesten von Martina Voigt in Bednarz, Die mittelalterlichen Glasmalereien in Berlin und Brandenburg (wie Anm. 47), S. 11-120 verwertet. Für die Berliner Pfarrkirchen sind solche Rechnungen erst aus der zweiten Hälfte des 16. Jahrhunderts erhalten, vgl. Christiane Schuchard, Die ältesten Rechnungsbücher der Berliner Pfarrkirchen St. Nikolai und St. Marien, in: Berlin in Geschichte und Gegenwart. Jahrbuch des Landesarchivs Berlin (2013), S. 7-60.

65 Auch für Sachsen sind solche Rechnungen aus vorreformatorischer Zeit selten. Eines der wenigen Zeugnisse behandelt Georg Buchwald, Das Rechnungsbuch der Kirche zu Niedersteinbach vom Jahre 1460 bis zur Reformation, in: Beiträge zur sächsischen Kirchengeschichte 32 (1919), S. 43-63.

66 Bernd Janowski/Dirk Schumann (Hg.), Dorfkirchen. Beiträge zu Architektur, Ausstattung und Denkmalpflege (Kirchen im ländlichen Raum 3), Berlin 2004.

67 Siehe oben bei Anm. 33, 34 und 36. 
Backstein (Dirk Schumann) im Kirchenbau der Mark Brandenburg, es werden kirchliche Bautypen in Berlin und der Mittelmark (Ulrich Waack) sowie im Preußenland (Christofer Herrmann) besprochen. Dendrodaten von Kirchen der Altmark (Ulf Frommhagen) und Dachwerke mittelalterlicher Dorfkirchen in Brandenburg (Tilo Schöfbeck) werden vorgestellt. Weiter geht es um Kirchen in Hinterpommern und der Neumark (Marek Ober) sowie Dorfkirchen in Mittelthüringen (Rainer Müller). Nur der Kunsthistoriker Dirk Höhne wendet sich aufgrund seiner Forschungen im Saalkreis exemplarisch den Schriftquellen zu: „Neuzeitliche Archivalien als Quellen zu mittelalterlichen Bauzuständen ländlicher Kirchen“ (S. 284-320) werden erörtert. Wie der Verfasser betont, sind die mittelalterlichen Quellen über Dorfkirchen allenthalben spärlich, aber auch die frühneuzeitliche Überlieferung bietet manche bislang ungenutzten Erkenntnismöglichkeiten über Bauzustände und Ausstattungsstücke. Der Themenbereich „Ausstattung“ umfasst fünf Lokal- und Regionalstudien: Putzfries an der Dorfkirche von Klaushagen (Jan Raue), Wandmalereien in der Kirche von Tremmen (Ute Joksch), die spätmittelalterliche Ausmalung in der Dorfkirche Altkalen in Mecklenburg (Dörte Brekenfeld), vorreformatorische Bildwerke in nachreformatorischen Altaraufsätzen (Werner Ziems) und Taufengel in der Uckermark (Beate Schroedter). Der Themenbereich „Funktions- und Nutzungsgeschichte“ wartet hingegen mit nur zwei Aufsätzen auf: Der Beitrag über romanische Landkirchen und ihre Ausstattung durch gotländische Stiftergemeinschaften (Tobias Kunz) fällt geografisch völlig aus dem Rahmen, bietet durch seine weitgehend hypothetischen Überlegungen aber auch keine brauchbaren Anhaltspunkte für die Erforschung der mitteldeutschen Kirchen. Ein wichtiger Bereich des pfarrlichen Lebens wird mit dem Beitrag über die „Dorfkirche als Begräbnisplatz“ (Blandine Wittkopp) angesprochen, doch bleibt mit den Beinhäusern ein wichtiger Aspekt spätmittelalterlicher Totenfürsorge ausgeklammert. ${ }^{68}$ Aus dem letzten Themenschwerpunkt des Dorfkirchenbandes, der mit „Denkmalpflege“ überschrieben ist, sollen zumindest die Beiträge über den Zustand der brandenburgischen Dorfkirchen (Roland Schneider) und über bürgerschaftliches Engagement zur Bewahrung denkmalgeschützter Kirchen (Bernd Janowski) erwähnt werden.

Als bisher letzter Band in der Reihe ist ein Sammelband über die Dorfkirchen in der Niederlausitz erschienen, der aber von mir bereits in dieser Zeitschrift besprochen wurde. ${ }^{69}$ Wie dort schon dargelegt, stehen Fragen der Baugeschichte, Archäologie, Ausstattung und Denkmalpflege im Vordergrund, während die historischen Dimensionen wieder zu kurz kommen. Unter der Überschrift „Zur Geschichte einer Landschaft“ äußern sich Ernst Badstübner zur Kunst- und Kulturlandschaft Niederlausitz, Bernd Janowski zur Devastierung der Dörfer im Lausitzer Braunkohlerevier, und nur mit Karlheinz Blaschke kommt ein Historiker zu Wort, der über „Kirchenpatrozinien und Kirchenorganisation in der Niederlausitz" (S. 1-17) freilich nichts Neues beizutragen vermag. ${ }^{70}$ Man vergleiche damit die Beiträge in einem neuen Tagungsband über die

68 Siehe dazu Enno Bünz, Memoria auf dem Dorf. Pfarrkirche, Friedhof und Beinhaus als Stätten bäuerlicher Erinnerungskultur im Spätmittelalter, in: Werner Rösener (Hg.), Tradition und Erinnerung in Adelsherrschaft und bäuerlicher Gesellschaft (Formen der Erinnerung 17), Göttingen 2003, S. 261-305, hier S. 292-300.

69 Vgl. oben Anm. 41.

70 Wie eigentlich immer in diesem Zusammenhang geht es um die Nikolaipatrozinien, worauf ich an dieser Stelle nicht weiter eingehen möchte. Es ist aber bezeichnend, dass diese Thesen beispielsweise von Friske, Barnim (wie Anm. 44), S. 443 oder Ders., Fläming (wie Anm. 45), S. 130 aufgegriffen werden, um bei Kirchen mit Nikolaipatrozinium über eine Marktfunktion des Ortes zu spekulieren. Das im Beitrag Blaschkes in 
Nieder- und Oberlausitz im Mittelalter, der eine umfangreiche Themensektion zur Christentumsgeschichte mit Bezügen auch zur Pfarrorganisation enthält. ${ }^{71}$

Die Umschau in der neueren Literatur über Dorfkirchen in Mitteldeutschland vermag der weiteren Forschung in Sachsen - sei es nun in Kritik oder Zustimmung - manche Anregungen zu geben.72 Wie deutlich wurde, befassen sich auf der einen Seite mittlerweile Kunsthistoriker, Bauforscher und Archäologen recht intensiv mit den mittelalterlichen Dorfkirchen, wie die vorgestellten Arbeiten vor allem über Brandenburg verdeutlichen. Auf der anderen Seite wird die Pfarrei mittlerweile von Historikern und Kirchenhistorikern intensiv erforscht, wie eingangs herausgestellt wurde. Ein Fazit der kritischen Sichtung neuerer Literatur lautet, dass unterschiedliche fachspezifische Forschungsstränge noch zu sehr nebeneinander herlaufen, zu wenig aufeinander abgestimmt sind. Die einen arbeiten über Pfarrkirchenbauten und ihre Ausstattung, die anderen über Pfarreiorganisation und das kirchliche Leben. ${ }^{73}$ Erst beide Perspektiven zusammen ergeben ein schlüssiges Gesamtbild. ${ }^{74} \mathrm{Da}$ in diesem Beitrag

Anm. 4 zitierte Werk von Karl Bosl über Regularkanoniker und Seelsorge (1979) ist völlig überholt. Bereits Stefan Weinfurter, Bemerkungen und Corrigenda zu Karl Bosls „Regularkanoniker und Seelsorge“, in: Archiv für Kulturgeschichte 62/63 (1981/82), S. 381-395, hat dazu alles Notwendige gesagt.

71 Heinz-Dieter Heimann/Klaus Neitmann/Uwe Tresp (Hg.), Die Nieder- und Oberlausitz. Konturen einer Integrationslandschaft, Bd. 1: Mittelalter (Studien zur brandenburgischen und vergleichenden Landesgeschichte 11), Berlin 2013, hier die Beiträge S. 39-188.

72 Wie mehrfach betont wurde, konnte im Rahmen dieser Besprechungsmiszelle kein bibliografischer Gesamtüberblick geboten werden. Hinzuweisen ist aber noch auf den recht guten Bearbeitungsstand für Thüringen, wo sich vor allem Rainer Müller, Mitarbeiter des Thüringischen Landesamtes für Denkmalpflege, große Verdienste um die Erforschung der Pfarrkirchen erworben hat: RaIner MüLler, Mittelalterliche Dorfkirchen in Thüringen, dargestellt anhand des Gebietes des ehemaligen Archidiakonats St. Marien zu Erfurt (Arbeitshefte des Thüringischen Landesamtes für Denkmalpflege, N. F. 2), Erfurt 2001 = Phil. Diss. Greifswald 2000; Ders., Die Kirchen in Neustadt an der Orla und Umgebung. Eine Geschichte des Sakralbaus in der Orlasenke (Beiträge zur Geschichte und Stadtkultur 18), Jena 2011; weiter zahlreiche Aufsätze dieses Autors.

73 Unverzichtbar ist der Beitrag der Liturgiegeschichte. Den Versuch, dass (katholische) gottesdienstliche Leben aus der Perspektive einer bestimmten Pfarrei zu erklären, unternehmen für Buxheim im Bistum Eichstätt Jürgen Bärsch/Johannes TrollMANN, Gottesdienstliches Leben in der Pfarrei Buxheim. Ein Führer zu Ausstattungsgegenständen der Liturgie in Vergangenheit und Gegenwart, Wemding 2008. Frömmigkeitsgeschichtlich bleibt das spätmittelalterliche Wallfahrtswesen von großer Faszinationskraft; zahlreiche wegweisende Studien zu mitteldeutschen Wallfahrten sind Hartmut Kühne zu verdanken, siehe z. B.: Auf Spurensuche: Wallfahrten zu Brandenburger Dorfkirchen im Spätmittelalter, in: Der Brandenburger Dom und die Dörfer, hrsg. vom Domstift Brandenburg, bearb. von Sigrid Philipps, Berlin 2004, S. 111-131; Hartmut Kühne/Anne-Katrin Ziesak (Hg.), Wunder - Wallfahrt - Widersacher. Die Wilsnackfahrt, Regensburg 2005; Felix Escher/Hartmut Kühne (Hg.), Die Wilsnack-Fahrt. Ein Wallfahrts- und Kommunikationszentrum Nord- und Mitteleuropas im Spätmittelalter (Europäische Wallfahrtsstudien 2), Frankfurt am Main u. a. 2006.

74 Vgl. exemplarisch Hartmut Kühne/Dirk Schumann, Die Wallfahrtskirche St. Annen in Alt Krüssow, Berlin 2006. - Mehrfach boten Jubiläen den Anlass, Stadtpfarrkirchen $\mathrm{zu}$ betrachten, wobei allerdings stadt- und kirchengeschichtliche, kunst- und baugeschichtliche Perspektiven unterschiedlich deutlich zur Geltung kamen, siehe z. B. 
vor allem von den Pfarrkirchen des Mittelalters die Rede war, muss abschließend noch eines betont werden: Die Pfarrkirchen stellen „Träger unserer ganzen Geschichte dar“, wie es Theodor Fontane (siehe das Eingangszitat) treffend formuliert hat. Wir betrachten mit ihnen Institutionen und Lebensformen, die vom Mittelalter bis in die Gegenwart reichen und damit epochenübergreifend Aufmerksamkeit verdienen. Die Pfarrei ist eben eine Einrichtung und eine Forschungsaufgabe von „langer Dauer“.75

Volker Leppin/Matthias Werner (Hg.), Inmitten der Stadt. St. Michael in Jena. Vergangenheit und Gegenwart einer Stadtkirche, Petersberg 2004; Franz Jäger (Hg.), Kirche in der Zeitenwende. Die Marktkirche Unser Lieben Frauen zu Halle in Spätmittelalter und Reformationszeit (Forschungen zur hallischen Stadtgeschichte 20), Halle 2013; Doreen Zerbe (Hg.), 800 Jahre St. Thomas zu Leipzig. Ein Gang durch die Geschichte, Leipzig 2014.

75 Petke, Die Pfarrei (wie Anm. 2), S. 17 f. 